\title{
A Test of the Collisional Dark Matter Hypothesis from Cluster Lensing
}

\author{
Jordi Miralda-Escudé ${ }^{1}$ \\ The Ohio State University, Dept. of Astronomy, McPherson Labs., 140 W. 18th Ave., \\ Columbus, $\mathrm{OH} 43210$ \\ jordi@astronomy. ohio-state.edu \\ ${ }^{1}$ Alfred P. Sloan Fellow
}

\begin{abstract}
Spergel \& Steinhardt proposed the possibility that the dark matter particles are self-interacting, as a solution to two discrepancies between the predictions of cold dark matter models and the observations: first, the observed dark matter distribution in some dwarf galaxies has large, constant-density cores, as opposed to the predicted central cusps; and second, small satellites of normal galaxies are much less abundant than predicted. The dark matter self-interaction would produce isothermal cores in halos and expel the dark matter particles from dwarfs orbiting in large halos. Another consequence of the model is that halos should become spherical once most particles have interacted. Several observations show that the mass distribution in relaxed clusters of galaxies is elliptical. Here, I discuss in particular gravitational lensing in the cluster MS2137-23, where the ellipticity of the dark matter distribution can be measured to a small radius, $r \sim 70 \mathrm{kpc}$, suggesting that most dark matter particles in clusters outside this radius do not collide during the characteristic age of clusters. If true, this implies that any dark matter self-interaction with a cross section independent of velocity is too weak to have affected the observed density profiles in the dark-matter dominated dwarf galaxies, or to have facilitated the destruction of dwarf satellites in galactic halos. If $s_{x}$ is the cross section and $m_{x}$ the mass of the dark matter particle, then $s_{x} / m_{x}<10^{-25.5} \mathrm{~cm}^{2} / \mathrm{GeV}$.
\end{abstract}

Subject headings: dark matter - galaxies: clusters: general - galaxies: formation - large-scale structure of universe 


\section{Introduction}

The Cold Dark Matter (CDM) model of structure formation in the universe has been tremendously successful in accounting for a huge variety of available observations (e.g., the Cosmic Background fluctuations, the abundances of clusters of galaxies, peculiar velocity fields, the Ly $\alpha$ forest), provided that the mean density of matter is only a fraction $\Omega_{m} \simeq$ 0.3 of the critical density, and the existence of vacuum energy with a negative pressure equation of state is allowed to make the universe spatially flat (e.g., (Knox \& Page 2000); (6); (Bahcall et al. 1999); (Strauss \& Willick 1995); (1); (Croft et al. 1999)).

A possible problem of this model has emerged when comparing the density profiles of dark matter halos predicted in numerical simulations, with observations of the rotation curves in dwarf galaxies ((Moore 1994); (Flores \& Primack 1994); (4); (Moore et al. 1998); (Kravtsov et al. 1998); (Moore et al. 1999b)). Whereas the observations show linearly rising rotation curves out to core radii greater than $1 \mathrm{kpc}$ in certain dwarf galaxies where the density is dominated by dark matter everywhere (indicating that the dark matter has a constant density core), the simulations predict that the collapse of collisionless particles of cold dark matter produces cuspy halo density profiles, with a logarithmic slope $-d \log \rho / d \log r>1$ down to the smallest resolved radius. A second problem is that the number of dwarf galaxies observed in the Local Group is much smaller than the total number predicted from numerical simulations ((Klypin et al. 1999); (Moore et al. 1999b)).

A solution to this discrepancy has been proposed by Spergel \& Steinhardt (2000): if the dark matter is self-interacting, with large enough cross section to make most particles in the inner core of a dwarf galaxy interact among themselves over a Hubble time, then an isothermal core will be produced. A clear prediction of this hypothesis is that when most of the particles of a halo within some radius $r_{c}$ have interacted, then the halo should be close to spherical inside $r_{c}$, or else be supported by rotation, because the velocity dispersion tensor should become isotropic. This paper examines the consequence of this prediction for the inner parts of rich clusters of galaxies, where highly magnified images of background galaxies are occasionally observed. We will find that severe restrictions on the collisional dark matter hypothesis are obtained.

\section{The Collisional Radius in Dwarf Galaxies and in Galaxy Clusters}

We assume that a halo of self-interacting dark matter has an initial density profile equal to the one for the case of collisionless dark matter, and is thereafter modified by the effects of the collisions. Numerical simulations of collisionless CDM models have shown that halos 
have a characteristic density profile, with a logarithmic slope that increases gradually with radius (Navarro, Frenk, \& White 1996, 1997; Moore et al. 1999b). We define the radius $r_{h}$ where the logarithmic slope is equal to 2 , so that $|d \log \rho / d \log r|<2$ at $r<r_{h}$, and $|d \log \rho / d \log r|>2$ at $r>r_{h}$. The particles closest to the center will be the first ones to collide, owing to the higher density. We define the collisional radius, $r_{c}$, as the radius within which more than half the particles have interacted. The effects of the collisions will be to change the velocity distribution of the particles inside the collisional radius toward a Maxwellian distribution, with constant velocity dispersion. This implies that the density profile within the collisional radius will be altered toward that of an isothermal sphere with finite core. The core radius produced by the collisions can obviously not be larger than the collisional radius, but it can be much smaller than the collisional radius if the initial slope of the halo profile inside $r_{c}$ was already close to isothermal, because the total energy needs to be conserved. Several numerical simulations have recently been done to model this effect (e.g., Burkert 2000, Yoshida et al. 2000, Davé et al. 2001).

In the initial density profile, the velocity dispersion should clearly decrease toward the center at $r<r_{h}$ : as long as the density profile has a central power-law cusp, and the orbits are not all highly radial near the center, then $\sigma^{2}(r) \propto \rho(r) r^{2}$. The collisions will therefore transport heat to the colder central particles from the hotter exterior, destroying the cusp and slowly increasing the core of the isothermal sphere as the collisional radius increases. However, the particles at $r>r_{h}$ should have a decreasing velocity dispersion with radius in their initial configuration, so when $r_{c}>r_{h}$ heat starts to be transported outward and the isothermal core shrinks as more particles are slung to the outer parts of the halo (or to unbound orbits), leading eventually to core collapse. As discussed by Spergel \& Steinhardt (2000), the cross section should be low enough so that the core collapse of the dark matter has not taken place in any halos up to the present time.

How should the collisional radius vary with the velocity dispersion of a dark matter halo? We assume that the cross section for the elastic collisions in the dark matter is independent of velocity, as expected in the low energy limit when the cross section is dominated by the s-wave contribution (e.g., Landau \& Lifshitz 1977). Then, the rate of interaction of a particle is proportional to the dark matter density, $\rho$, times the velocity dispersion $\sigma$. Hence, $\rho \sigma t=$ constant, where $t$ is the age of the halo (or the time since the last merger which determined an initial density profile). Assuming that the core of the halo is not larger than the collisional radius, dynamical equilibrium implies $\rho\left(r_{c}\right) \propto \sigma^{2} / r_{c}^{2}$, and therefore,

$$
r_{c} \propto \sigma^{3 / 2} t^{1 / 2}
$$

This implies that if the core radii in dwarf galaxies are caused by dark matter collisions within a larger collisional radius, then all the galactic and cluster dark matter halos should 
have much larger collisional radii as their velocity dispersion increases.

Typically, the constant density cores of dwarf galaxy halos measured from the kinematics of the HI gas extend out to a few $\mathrm{kpc}$, and a typical velocity dispersion is $50 \mathrm{~km} \mathrm{~s}^{-1}$. As a few examples, the rotation curves of the dwarfs DDO 154, DDO 170, and DDO 236 yield fits for their dark matter halos with velocity dispersion $\sigma=(28,52,45) \mathrm{km} \mathrm{s}^{-1}$, and core radii $(3,2.5,6)$ kpc ((Carignan \& Beaulieu 1989); (2); (Jobin \& Carignan 1990)), with assumed distances of $(4,15,1.7) \mathrm{Mpc}$, respectively.

If we wish to explain the sizes of these dark matter cores in dwarf galaxies as the result of collisional dark matter, then the collisional radii of the halos of these dwarfs must be larger than the observed core radii, and the collisional radii in rich clusters of galaxies must be much larger, according to (1). Using the conservative values of $r_{c}=2 \mathrm{kpc}$ and $\sigma=50 \mathrm{~km} \mathrm{~s}^{-1}$ for a typical dwarf galaxy, and assuming that a typical rich cluster is about a third as old as a dwarf galaxy (since massive halos have collapsed more recently than dwarf galaxies; see Fig. 10 of Lacey \& Cole 1993), we infer that the collisional radius of a typical rich cluster with velocity dispersion $\sigma=1000 \mathrm{~km} \mathrm{~s}^{-1}$ should be at least $r_{c}>100 \mathrm{kpc}$.

Within the collisional radius, the halo potential should be very nearly spherical because the collisions should make the velocity dispersion tensor of the dark matter particles isotropic (unless the core is rapidly rotating, which is highly unlikely as will be discussed in $\S 4$ ). This is most easily seen for a finite system, using the tensor virial theorem: the potential energy tensor (which reflects the shape of the mass distribution) will become diagonal over the same timescale as the kinetic energy tensor. The next section discusses the evidence from gravitational lensing showing that cluster cores are elliptical in their inner parts, focusing in particular on the example of MS2137-23.

\section{The core of the cluster MS2137-23 is elliptical}

Highly magnified images of background galaxies (or "arcs") produced by gravitational lensing have been observed in many clusters of galaxies. In general, models that reproduce the positions and shapes of these images assume the presence of elliptical clumps of dark mat-

ter centered on the most luminous galaxies in the cluster, with the ellipticity being oriented along the same axis as the optical light. Examples of clusters that have been modeled in this way include A370 ((Kneib et al. 1993)), A2218 ((Kneib et al. 1995)), MS2137-23 ((3)), and A2390 ((Pierre et al. 1996)). It should be noted that the optical isophotes of the central cluster galaxies generally extend out to the radius where the gravitationally lensed images are observed, where the potential is strongly dominated by the dark matter. The regular 
elliptical isophotes of the distribution of stars implies that the gravitational potential has the same shape, and this is confirmed by the lensing models that reproduce the positions and shapes of the multiple images of background galaxies.

We note here the intriguing fact that the isophotes of central cluster galaxies tend to show a decrease of the ellipticity toward the center, within radii $\lesssim 10 \mathrm{kpc}$ (Porter et al. 1991). This might plausibly be an indication of the effects of self-interacting dark matter at this small radius, making the potential more spherical; however, other dynamical effects associated with the formation of these galaxies from mergers might also explain this if the dark matter is collisionless. In this paper, we will discuss the evidence that if there is selfinteracting dark matter, the collisional radius in rich clusters of galaxies should be smaller than $\sim 100 \mathrm{kpc}$, leaving the question of whether there might a smaller collisional radius for future work.

Here, we shall focus on the cluster MS2137-23. This cluster has several characteristics that make it particularly useful for our purpose. First, the central region of the cluster appears to be well relaxed as shown from both the optical image, dominated by the central galaxy, and the X-ray emission, centered on the galaxy and with an ellipticity and position angle similar to that of the central galaxy ((Hammer et al. 1997)). In clusters with substructure, the presence of multiple mass clumps requires models of the mass distribution with many parameters, making it difficult to constrain the ellipticity of each mass clump. Second, a total of five gravitationally lensed images arising from two sources are observed in MS2137-23, providing many constraints for the lensing model. Although redshifts for these five images have not yet been measured, their morphologies and colors provide strong evidence for the lensing interpretation ((Hammer et al. 1997)). One source produces a long, tangential arc and two other arclets, and the second source gives rise to a radially elongated image near the center and another arclet (where "arclet" refers to images that are not magnified by very large factors, but still show a characteristic stretching effect due to lensing).

The positions and relative sizes and shapes of these five images can be reproduced in an extremely simple model: an elliptical mass clump centered on the central galaxy, with the same ellipticity and position angle ((3); (Miralda-Escudé 1995)). This model needs only two free parameters for the radial density profile (the velocity dispersion of the cluster and the core radius). Since the positions of the five images alone already provide 6 constraints (ten coordinates of the five images minus 4 for the unknown positions of the two sources), and in addition the relative sizes and orientations of each image are also reproduced, this should be considered as strong evidence that the potential of the dark matter is elliptical, just like the stellar isophotes, and has not been significantly circularized by dark matter collisions at 
the radius where the images are observed. This radius is 15 " for the longest tangential arc, which corresponds to $70 \mathrm{kpc}$ (for $H_{0}=70 \mathrm{~km} \mathrm{~s}^{-1} \mathrm{Mpc}^{-1}$ ). The radially elongated image is only 5" from the cluster center; however, if the potential became spherical only at this small radius, this radial image would not be significantly altered.

Could other perturbations to the potential, arising from substructure (which causes external shear), mimic the effect of ellipticity if the true potential was spherical within $\sim 100 \mathrm{kpc}$ ? There are two arguments against this possibility. First, an external shear would be roughly constant within the region of the multiple images, whereas an elliptical potential causes a variable shear and convergence that depend on the density profile (see eqs. 2 to 8 below). Second, there would be no reason why the external shear should be aligned with the major axis of the galaxy. While substructure is common in many clusters, the central parts of MS2137-23 appear relaxed, as discussed above.

Although the fact that the simple elliptical potential, with constant ellipticity as a function of radius, fits the observed positions and shapes of the five images can already be considered as persuasive evidence that the potential cannot be spherical within $\sim 100 \mathrm{kpc}$, it will be useful to show analytically why an ellipticity is required in a model-independent manner. We will focus here on the radial image and its counterimage. These two images of the same source are labeled as A1 and A5 in Mellier et al. (1993), and in Figure 1 of Miralda-Escudé (1995), and as AR and A5 in the HST image presented in Hammer et al. (1997).

A schematic representation of the lensing of the source on the radial caustic is shown in Figure 1, which defines the notation that will be used here. The point labeled $C$ is the center of the cluster, and $S$ is the position of the source that gives rise to the radial image at $R$ and the counterimage at $I$ (the entire lensing configuration in this system, with the critical lines and caustics of a simple elliptical potential, is shown in Fig. 1 of Miralda-Escudé 1995). We use polar coordinates on the image plane: $\theta$, the angular distance from the center $C$, and $\phi$, the azimuthal angle. The light ray observed at $R$ is deflected by an angle $\alpha_{\theta R}$ in the radial direction, and $\alpha_{\phi R}$ in the azimuthal direction, and the same for the light ray observed at $I$.

The specific observed quantity that we will relate to the ellipticity of the potential is the angle $\gamma$ of misalignment between the images $R$ and $I$, relative to the center of the lens. In a spherical potential, the images $R$ and $I$ should lie on a straight line passing through $C$. The observed angle is $\gamma=19^{\circ}$, indicating that the potential is elliptical. In principle, this misalignment could also be caused by substructure in the cluster, but this is unlikely in view of the relaxed appearance of the cluster.

We now relate the angle $\gamma$ to the ellipticity and the density profile of the potential. If the 


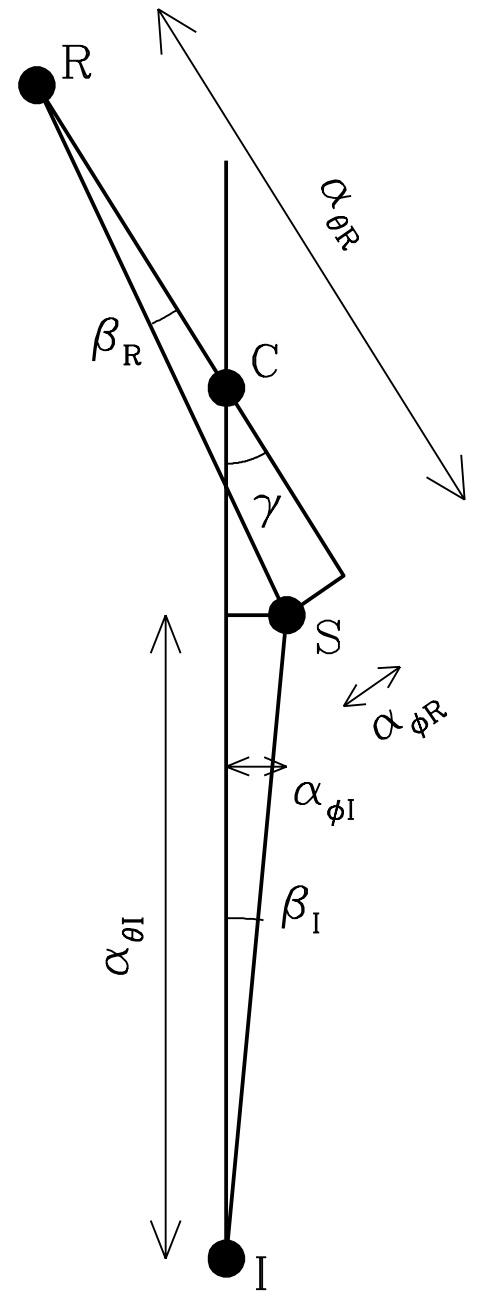

Fig. 1.- Schematic representation of the lensing configuration in the cluster MS2137-23 discussed in $\S 3$. The lens center is at $C$, the source is located at $S$, and its two images are observed at $R$ (which is the image on the radial critical line) and $I$. The angle of misalignment $\gamma$ between the two images relative to the center would be zero for a spherical potential. The radial and azimuthal components of the deflection angles $\left(\alpha_{\theta}\right.$ and $\left.\alpha_{\phi}\right)$ are indicated. 
ellipticity $\epsilon$ is small, the projected potential is adequately approximated with a quadrupole term (e.g., (Miralda-Escudé 1995)),

$$
\psi(\theta, \phi)=\psi_{0}(\theta)-\frac{\epsilon}{2} \psi_{1}(\theta) \cos (2 \phi),
$$

where

$$
\begin{gathered}
\psi_{0}(\theta)=\int_{0}^{\theta} d \theta^{\prime} \alpha_{0}\left(\theta^{\prime}\right), \\
\alpha_{0}(\theta)=\frac{2}{\theta} \int_{0}^{\theta^{\prime}} d \theta^{\prime} \theta^{\prime} \kappa_{0}\left(\theta^{\prime}\right) \equiv \theta \bar{\kappa}_{0}\left(\theta^{\prime}\right), \\
\psi_{1}(\theta)=\frac{2}{\theta^{2}} \int_{0}^{\theta} d \theta^{\prime} \theta^{\prime 3} \kappa_{0}\left(\theta^{\prime}\right),
\end{gathered}
$$

and where the surface density of the lens is

$$
\kappa(\theta, \phi)=\kappa_{0}(\theta)-\frac{\epsilon}{2} \theta \frac{d \kappa_{0}}{d \theta} \cos (2 \phi) .
$$

Here, $\kappa_{0}(\theta)$ is the azimuthally averaged surface density profile, and $\bar{\kappa}_{0}(\theta)$ is the averaged surface density within $\theta$. The deflection angle is given by the gradient of the potential,

$$
\begin{gathered}
\alpha_{\theta}=\theta \bar{\kappa}_{0}(\theta)+\theta\left[\kappa_{0}(\theta)+\frac{\psi_{1}(\theta)}{\theta^{2}}\right] \epsilon \cos (2 \phi), \\
\alpha_{\phi}=\frac{\psi_{1}(\theta)}{\theta} \epsilon \sin (2 \phi) .
\end{gathered}
$$

In the limit of a small ellipticity of the potential, the angle of misalignment $\gamma$ is given by (using the notation in Fig. 1),

$$
\gamma=\beta_{I} \frac{\alpha_{\theta I}}{\theta_{I}-\alpha_{\theta I}}+\beta_{R} \frac{\alpha_{\theta R}}{\alpha_{\theta R}-\theta_{R}}=\frac{\alpha_{\phi I}}{\theta_{I}-\alpha_{\theta I}}+\frac{\alpha_{\phi R}}{\alpha_{\theta R}-\theta_{R}} .
$$

Using the condition that the rays at images $R$ and $I$ are deflected to the same position $S$, which is simply $\theta_{i}-\alpha_{\theta I}=\alpha_{\theta R}-\theta_{R}$ (the ellipticity introduces only second order corrections here), we obtain

$$
\gamma=\left[\frac{\psi_{1}\left(\theta_{R}\right)}{\theta_{R}}+\frac{\psi_{1}\left(\theta_{I}\right)}{\theta_{I}}\right] \frac{\epsilon \sin \left(2 \phi_{I}\right)}{\theta_{I}-\alpha_{\theta I}} .
$$

We now want to find a lower limit to the ellipticity necessary to generate the observed angle $\gamma$. For this purpose, it will be convenient to replace the function $\psi_{1}(\theta) / \theta$ ) by an upper limit. Using equation (5), we find that if the $\kappa_{0}$ is constant within $\theta$, then $\psi_{1}(\theta) / \theta=$ $\theta \bar{\kappa}_{0}(\theta) / 2$, while in any profile where $\kappa_{0}$ decreases with radius, we have $\psi_{1}(\theta) / \theta<\theta \bar{\kappa}_{0}(\theta) / 2$, 
because the integral of equation (5) weights more heavily the surface density near $\theta$ than at smaller angular radius. Therefore,

$$
\gamma<\frac{\left[\theta_{R} \bar{\kappa}_{0}\left(\theta_{R}\right)+\theta_{I} \bar{\kappa}_{0}\left(\theta_{I}\right)\right] \epsilon \sin \left(2 \phi_{I}\right)}{2 \theta_{I}\left[1-\bar{\kappa}\left(\theta_{I}\right)\right]}=\frac{\left(1+\theta_{R} / \theta_{I}\right) \epsilon \sin \left(2 \phi_{I}\right)}{2\left[1-\bar{\kappa}\left(\theta_{I}\right)\right]} .
$$

We can now substitute the observed values $\theta_{I}=22^{\prime \prime} .5$ ((Fort et al. 1992)), and $\theta_{R}=5^{\prime \prime} .2$ ((Hammer et al. 1997)):

$$
\gamma<0.62 \frac{\epsilon \cos \left(2 \phi_{I}\right)}{1-\bar{\kappa}_{0}\left(\theta_{I}\right)} .
$$

To obtain a lower limit to $\epsilon$, we need to assume an upper limit for $1-\bar{\kappa}\left(\theta_{I}\right)$. Because the two images $R$ and $I$ result from radial (rather than tangential) magnification, there is no reason why $\bar{\kappa}$ needs to be particularly close to unity at either image. Given the relation $\left[\bar{\kappa}_{0}\left(\theta_{R}\right)-1\right] /\left[1-\bar{\kappa}_{0}\left(\theta_{I}\right)\right]=\theta_{I} / \theta_{R}=4.3$, the quantity $1-\bar{\kappa}\left(\theta_{I}\right)$ could be very small only if the surface density profile was very flat between the angular radii $\theta_{R}$ and $\theta_{I}$. This is very unlikely because the velocity dispersion implied for the cluster for an Einstein radius close to $\theta_{I}=22^{\prime \prime} .5$ is already larger than $1000 \mathrm{~km} \mathrm{~s}^{-1}$ (see (Miralda-Escudé 1995), Figs. 8 and 9), and it would increase to a much higher value at large radius if the slope of the density profile was much shallower than isothermal at $\theta \sim \theta_{I}$.

As a reasonable limit on how flat the $\bar{\kappa}$ profile could be from $\theta_{R}$ to $\theta_{I}$, we will assume here $\bar{\kappa}\left(\theta_{R}\right) / \bar{\kappa}\left(\theta_{I}\right)>2$ (remember that $\theta_{I} / \theta_{R}=4.3$ ). This corresponds to $1-\bar{\kappa}_{0}\left(\theta_{I}\right)>0.16$, implying that the image $I$ is not tangentially magnified by more than a factor 6 , which is reasonable given the length of the image $I$ (called A5 in (Hammer et al. 1997)), $\sim 3^{\prime \prime}$, and its axis ratio of $\sim 3$.

With this condition, and using also $\cos \left(2 \phi_{I}\right) \simeq 0.7$ (e.g., Mellier et al. 1993; we assume the major axis of the potential is aligned with that of the central galaxy), and $\gamma=0.33$, the lower limit on the ellipticity from equation (12) is

$$
\epsilon>0.77\left[1-\bar{\kappa}\left(\theta_{I}\right)\right] \gtrsim 0.1 .
$$

This is only a lower limit that we have obtained using only one observational constraint, the misalignment of two images relative to the center. The models that reproduce also the three images of the other source require an ellipticity $\epsilon \simeq 0.2$.

There are other clusters that show little substructure in their inner parts and are well modelled by an elliptical potential with the major axis coinciding with that of the central galaxy: one is A2218 (Kneib et al. 1995), which requires two clumps in the model, but with the dominant agreeing in position and ellipticity with the central cluster galaxy. Another is A963, which shows two tangential arcs around the central giant elliptical (Lavery \& Henry 
1988). In the case of A963 the ellipticity is difficult to constrain because there are only two images which could be from the same source or two different sources.

\section{Discussion}

The modeling of multiple images of background galaxies produced by gravitational lensing in clusters of galaxies require elliptical models of the mass distribution in order to reproduce their positions and magnifications successfully ((Kneib et al. 1993); (3); (Kneib et al. 1995)). The last section discussed the specific example of MS2137-23, where the misalignment in the position of two images relative to the cluster center can be used to constrain the ellipticity in a model-independent way: the ellipticity of the dark matter halo around the central galaxy must be greater than 0.1 within the image $I$, which is at $22^{\prime \prime} .5$ from the cluster center, corresponding to a distance of $65 h^{-1} \mathrm{kpc}$. The fact that the dark matter halos of galaxy clusters are elliptical within this small radius implies that the dark matter particles have not collided over the age of the cluster. As shown in $\S 2$, this also implies that the observed cores of the dark matter halos in dwarf galaxies are too big to have been caused by dark matter self-interaction, as proposed by Spergel \& Steinhardt (2000).

Further evidence supporting that cluster dark matter halos are elliptical at radii $\sim 100$ kpc comes from the similarity with the ellipticity of the optical isophotes of the central cluster galaxies in both the magnitude of the ellipticity and the orientation of the major axis ((3); (Kneib et al. 1993); (Kneib et al. 1995)). If the underlying dark matter distribution became spherical due to the collisions, the ellipticity of the stellar distribution would be reduced (although not eliminated, owing to the anisotropy in the velocity dispersion tensor). According to Hammer et al. (1997), the central galaxy in MS2137-23 has ellipticity $\epsilon=$ $0.16 \pm 0.02$ beyond the radius of the radial arc, and the best fit ellipticity for the lens model is $\epsilon=0.18$ (see also (Kneib et al. 1995) for similar conclusions obtained in the cluster A2218). We note again that the ellipticities of the optical isophotes decline at a radius smaller than that probed by gravitational lensing (Porter et al. 1991).

The ellipticity of the cluster halo can be used to place an upper limit on the interaction rate of the dark matter, in terms of the cross section $s_{x}$ and mass $m_{x}$ of the dark matter particle. We assume here that the collisional radius must be smaller than the distance from the center to the long tangential arc and two other arclets (these images are A01-A02, A2 and A4 in (Hammer et al. 1997), and they also require an ellipticity similar to that of the central galaxy in the lensing models), which is about $70 \mathrm{kpc}$. The dark matter density at this radius is $\rho \simeq \Sigma_{\text {crit }} / 2 r$, where the critical surface density is $\Sigma_{\text {crit }} \simeq 1 \mathrm{~g} \mathrm{~cm}^{-2}$ for a source at $z_{s}=1$. Assuming also a cluster velocity dispersion $\sigma=1000 \mathrm{~km} \mathrm{~s}^{-1}$ (roughly the minimum 
value required given the Einstein radius of the cluster), and a cluster age $t_{c}=5 \times 10^{9}$ years, we obtain the upper limit

$$
\frac{s_{x}}{m_{x}}<\frac{1}{\rho 2^{1 / 2} \sigma t_{c}} \simeq 10^{-25.5} \frac{\mathrm{cm}^{2}}{m_{p}} \simeq 0.02 \frac{\mathrm{cm}^{2}}{\mathrm{~g}} .
$$

For the dwarf galaxies DDO 154, DDO 170, and DDO 236 mentioned in $\S 2$, with velocity dispersion $\sigma=(28,52,45) \mathrm{km} \mathrm{s}^{-1}$, and core radii $(3,2.5,6) \mathrm{kpc}$, the time it would take for the collisional radius to reach the value of their core radii if $s_{x} / m_{x}$ were equal to the above upper limit is $t=(40,5,40) \times 10^{10}$ years, respectively [where we have used the relation $t \propto \sigma^{3} / r_{c}^{2}$, from eq. (1) ].

The limit we have obtained on the self-interaction of the dark matter also rules it out as an explanation for the low abundance of dwarf galaxies in the Local Group, compared to the predictions of halo satellites abundances from numerical simulations ((Klypin et al. 1999); (Moore et al. 1999a)). In order to strike out the dark matter particles, the satellite halos must be moving in an orbit inside the collisional radius. For example, in the Milky Way halo (with $\sigma \simeq 150 \mathrm{~km} \mathrm{~s}^{-1}$ ), the collisional radius cannot be greater than about $6 \mathrm{kpc}$, if $r_{c}<100 \mathrm{kpc}$ in a cluster with $\sigma=1000 \mathrm{~km} \mathrm{~s}^{-1}$ (where we use the scaling $r_{c} \propto \sigma^{3 / 2}$ ).

Finally, we mention three ways by which the collisional dark matter hypothesis might still remain viable as an explanation of the constant density cores observed in some dwarf galaxies. A first possibility is that the presence of substructure in the mass distribution of MS2137-23, or of other massive structures projected on the line of sight of the cluster, introduces an external shear that would modify the positions of the images. However, this seems unlikely as discussed in $\S 3$, because elliptical models fit the observed positions and shapes of the images remarkably well with fewer model parameters than observational constraints, and an external shear induces a lensing potential different than a constant ellipticity, and would not generally be aligned with the major axis of the galaxy. The second possibility is that the ellipticity of the dark matter could be supported by rotation, instead of anisotropic velocity dispersion. However, halos formed by collisionless collapse are known to rotate very slowly ((Barnes \& Efstathiou 1987); (Warren et al. 1992)), and the collisions would further slow down the rotation of the central parts of the halo by enforcing solid body rotation. Finally, there is the possibility that the cross section for the dark matter interaction decreases with velocity. Here we have assumed the cross section to be constant; if it were proportional to $v^{-1}$ (see, e.g., Firmani et al. 2000), then the constraints we have used here from gravitational lensing in clusters of galaxies would allow a large enough collisional radius in dwarfs to explain their dark matter core radii.

I am grateful to Andy Gould, Paul Steinhardt and David Weinberg for discussions and 
for their encouragement. 


\section{REFERENCES}

Bahcall, N., Ostriker, J. P., Perlmutter, S., \& Steinhardt, P. J. 1999, Science, 284, 1481

Barnes, J., \& Efstathiou, G. 1987, ApJ, 319, 575

Burkert, A. 2000, ApJ, 534, L143

Carignan, C., \& Beaulieu, S. 1989, ApJ, 347, 760

Croft, R. A. C., Weinberg, D. H., Pettini, M., Hernquist, L., \& Katz, N. 1999, ApJ, 520, 1

Davé, R., Spergel, D. N., Steinhardt, P. J., \& Wandelt, B. D. 2001, ApJ, 547, 574

Eke, V. R., Cole, S., \& Frenk, C. S, 1996, MNRAS, 282, 263

Firmani, C., D’Onghia, E., Chincarini, G., Hernández, X., \& Avila-Reese, V. 2001, MNRAS, 321,723

Flores, R. A., \& Primack, J. A. 1994, ApJ, 427, L1

Fort, B., Le Fèvre, O., Hammer, F., \& Cailloux, M. 1992, ApJ, 399, L125

Hammer, F., Gioia, I. M., Shaya, E. J., Teyssandier, P., Le Fèvre, O., \& Luppino, G. A. 1997, ApJ, 491, 477

Jobin, M., \& Carignan, C. 1990, AJ, 100, 648

Klypin, A. A., Kravtsov, A. V., Valenzuela, O., \& Prada, F. 1999, ApJ, 522, 82

Kneib, J. P., Mellier, Y., Fort, B., \& Mathez, G. 1993, A\& A, 273, 367

Kneib, J. P., Mellier, Y., Pelló, R., Miralda-Escudé, J., Le Borgne, J.-F., Böhringer, H., \& Picat, J.-P. 1995, A\&A, 303, 27

Knox, L., \& Page, L. 2000, Phys. Rev. Lett., 85, 1366

Kravtsov, A. V., Klypin, A. A., Bullock, J. S., \& Primack, J. R. 1998, ApJ, 502, 48

Lacey, C., \& Cole, S. 1993, MNRAS, 262. 627

Lake, G., Schommer, R. A., \& van Gorkom, J. A. 1990, AJ, 99. 547

Landau, L. D., \& Lifshitz, E. M. 1977, Quantum Mechanics (Non-Relatistic Theory) (Pergamon Press). 
Lavery, R. J., \& Henry, J. P. 1988, ApJ, 329, L21

Mellier, Y., Fort, B., \& Kneib, J. P. 1993, ApJ, 407, 33

Miralda-Escudé, J. 1995, ApJ, 438, 514

Moore, B. 1994, Nat, 370, 629

Moore, B., Governato, F., Quinn, T., Stadel, J., \& Lake, G. 1998, ApJ, 499, L5

Moore, B., Ghigna, S., Governato, F., Lake, G., Quinn, T., Stadel, J., \& Tozzi, P. 1999, ApJ, 524, L19 (astro-ph/9907411)

Moore, B., Quinn, T., Governato, F., Stadel, J., \& Lake, G. 1999, MNRAS, 310, 1147

Navarro, J. F., Frenk, C. S., \& White, S. D. M. 1996, ApJ, 462, 563

Navarro, J. F., Frenk, C. S., \& White, S. D. M. 1997, ApJ, 490, 493

Perlmutter, S., Turner, M. S., \& White, M. 1999, Phys. Rev. Lett., 83, 670

Pierre, M., Le Borgne, J. F., Soucail, G., \& Kneib, J. P. 1996, A\& A, 311, 413

Porter, A. C., Schneider, D. P., \& Hoessel, J. G. 1991, AJ, 101, 1561

Spergel, D. N., \& Steinhardt, P. J. 1999, Phys. Rev. Lett. 84, 3760

Strauss, M. A., \& Willick, J. A. 1995, Phys. Rep., 261, 271

Warren, M. S., Quinn, P. J., Salmon, J. K., \& Zurek, W. H. 1992, ApJ 399, 405

Yoshida, N., Springel, V., White, S. D. M., \& Tormen, G. 2000, ApJ, 544, L87 